\title{
Deaths of HIV-Positive Men in the Context of Assisted Reproduction: Five Case Studies from a Single Center
}

\author{
GARY S. NAKHUDA, M.D., JOSEPH E. PENA, M.D., and MARK V. SAUER, M.D.
}

\begin{abstract}
Provision of reproductive services to individuals infected with HIV-1 is gaining popular acceptance and is generally endorsed by specialists in reproductive medicine. In the situation in which the male is HIV positive and the female partner is not infected, a large body of evidence has demonstrated that the use of assisted reproductive technology is effective for achieving pregnancy, while eliminating the risk of viral transmission to the mother and fetus. No reports have documented the well-being of the HIV-infected partners subsequent to seeking fertility services. In the current report, we document the cases of five HIV-positive men who died secondary to complications of HIV infection shortly after participating in the assisted reproduction program for HIV-1-serodiscordant couples at Columbia University. Three of these couples successfully achieved pregnancy and live birth, including one set of triplets, and one case of posthumous conception; the fourth case resulted in the cryopreservation of all embryos after the sudden death of the male before the time of embryo transfer; the fifth couple failed to conceive. None of the deaths, which occurred within a few months to 2 years from initial consultation, were related to infertility treatment. The demographic and social statuses of these patients were not different from the general population of men seeking assisted reproduction in our clinic. Regarding the HIV infection status of these cases, three patients had a longer duration of infection compared to the general population of men in our cohort, and one had a significantly lower CD4 cell count. All five men had stable HIV viral loads, and were determined by their primary care providers to be clinically healthy at the time of entry into the program for assisted reproduction. The untimely deaths of these patients underscores the importance of the thoughtful consideration of the complex issues involved in family planning for these individuals, including advanced directives for the use of cryopreserved gametes and embryos, and the social, emotional, and practical issues for the children and surviving partners subsequent to the death of the HIV-positive parent.
\end{abstract}

\section{INTRODUCTION}

$\mathbf{T}$ he American College of Obstetrics \& GyNECOLOGY and the American Society for Reproductive Medicine endorse the use of assisted reproductive technologies for men, women, and couples infected with HIV-1., ${ }^{1,2}$ These recommendations represent revisions of previous opinions expressed by these agencies and others, ${ }^{3-8}$ which were not generally in favor of the use of assisted reproduction for couples with HIV-1 serodiscordance. The changes

Columbia University College of Physicians and Surgeons, Department of Obstetrics and Gynecology, New York, New York. 
in policies were at least partly influenced by the dramatic improvement in survival of individuals infected with HIV-1, largely because of the development of highly active antiretroviral therapy (HAART). With the reduction in AIDS mortality, ${ }^{9}$ and the prolonged interval from infection to death, ${ }^{10,11}$ it is reasonable to assume that many HIV-1-seropositive patients of reproductive age will consider having children. In a survey of 1440 patients with HIV, 29\% expressed desire for children, ${ }^{12}$ and many serodiscordant couples reject the notion of using donor sperm for conception. ${ }^{13}$

HIV-1-serodiscordant couples now have the opportunity to seek assisted reproduction for infertility and minimization of viral transmission. The largest experience with assisted reproduction in couples with HIV-1 serodiscordance in which the male is infected, comes from the collective European database, where since 1989, more than 500 infants have been born after nearly 5000 cycles of assisted reproduction in 7 countries without a single report of maternal or perinatal transmission. ${ }^{14}$ In the United States, $18 \%$ of 182 fertility clinics that responded to a recent survey reported providing some form of assisted reproduction to HIV1-infected couples, ${ }^{15}$ however, the extent of services provided was not specified. In our clinic, since 1997 we have performed over 250 cycles of in vitro fertilization with intracytoplasmic sperm injection (IVF-ICSI) on 130 serodiscordant couples of whom the male is HIV-positive, yielding more than 100 babies without a single maternal or perinatal infection. As the prevalence of HIV-1 continues to grow in reproductive-aged patients, ${ }^{9}$ awareness and utilization of such services is certain to increase.

While the data favors optimism toward providing reproductive care to HIV-1-serodiscordant couples, there have been few reports detailing the lives of these individuals after their participation in assisted reproduction programs. In our program, every effort is made to ensure that HIV-1 testing is repeated 6 months postpartum in mothers and infants in order to verify that periconceptual and perinatal transmission has not occurred. Care of the infected parent, however, is the onus of the infectious disease specialist, and there is generally a lack of data in the reproductive medicine literature concerning the short- and long-term outcomes of treated patients. Death or incapacitation of a seropositive patient after successful assisted reproduction will undoubtedly have a profound impact on the surviving partner and offspring.

In the current report, we describe five cases in which the HIV-1-seropositive male partner died within a short time after participating in assisted reproduction. The deaths occurred several months to 2 years after the initial consult, and none of the fatalities were related to infertility treatment. Three of the five couples achieved live births including one set of triplets, and one conception that was initiated by frozen embryo transfer (FET) after the death of the HIV-positive male partner. In the other two cases, one woman cryopreserved all of her embryos after the death of her husband, while the other couple failed to achieve pregnancy and had no cryopreserved embryos for later transfer. While these patients represent a minority of our HIV-1-serodiscordant cohort, their deaths emphasize the need to provide thoughtful counseling to patients who are otherwise favorable candidates for assisted reproduction.

\section{MATERIALS AND METHODS}

The protocol for offering IVF-ICSI to HIV1-serodiscordant couples in which the male is infected was approved by the Institutional Review Board (IRB) and the Ethics Committee of Columbia University. We retrospectively reviewed the charts of 191 couples who presented for consultation for the IVF-ICSI program for HIV-serodiscordant couples at Columbia University between August 1997 and December 2004. One-hundred thirty couples met criteria for enrollment and participated in the protocol. Serodiscordant couples in which the female is HIV-positive are treated under a similar IRB approved protocol but were not included in the current data. Enrollment criteria into our is described elsewhere. ${ }^{16}$ Briefly, HIV1-seropositive men were required to be in good health, without active AIDS-defining illness, verified by a letter of clearance from their in- 
fectious disease specialist. Treatment with antiretrovirals was per discretion of the primary care providers. Viral load (HIV-RNA-PCR) was required to be less than 30,000 copies per milliliter, and stable over 6 months. CD4 ${ }^{+}$T-cell counts also must have demonstrated stability over the 6 months prior to treatment. Female partners were required to demonstrate HIV-1 seronegativity using an enzyme immunoassay (HIV-EIA) screen within 6 months of initiating therapy. The long-term practice of safe sex with condoms was ascertained at interview. A general health and reproductive screening was performed on couples prior to attempting assisted reproduction, including serological testing for hepatitis B and C. Hepatitis viremia does not contraindicate IVF. ${ }^{17}$ Counseling by specialists in Maternal Fetal Medicine and Psychiatry or Social Services was encouraged and readily available. Informed consent prior to each cycle included an advanced directive regarding the fate of cryopreserved embryos and semen in the event of the patient(s) death.

The processing of sperm was performed as previously described. ${ }^{16}$ Handling of semen samples from HIV-seropositive men was performed in a separate class II biologic safety cabinet using strict universal precautions. The semen was centrifuged through a discontinuous density gradient (Sage BioPharma, Bedminster, NJ). The resulting pellet was transferred to a clean conical tube and resuspended in modified human tubal fluid (HTF) (Irvine Scientific, Santa Ana, CA) supplemented with 5\% human serum albumin (HSA) (Sage BioPharma). The sperm were centrifuged for a maximum of 10 minutes at $300 g$, and the supernatant (wash number 1) was removed. The pellet was resuspended in $3 \mathrm{~mL}$ of fresh modified HTF-HSA and spun again for a maximum of 5 minutes. The supernatant (wash number 2) was removed, and the pellet was resuspended in a small volume of modified HTF-HSA for swimup. The purified sperm were counted, and the concentration was lowered, if necessary, to approximately 5 to $10 \times 10^{6}$ motile sperm per milliliter (final sperm preparation). IVF-ICSI, the process of individually fertilizing retrieved oocytes by the injection of single spermatozoa, was performed according to established protocol. ${ }^{18}$

Ongoing pregnancies were followed by Maternal Fetal Medicine in each trimester, and HIV-RNA-PCR was performed on pregnant women every 3 months during the pregnancy. Infants and mothers were tested by either HIVRNA-PCR or HIV-DNA-PCR at birth and 3 and 6 months later. In nonpregnant patients, HIVEIA screens were performed 3 months after embryo transfer. Patients were contacted annually thereafter for additional follow-up, by telephone or by mail when unreachable by telephone.

\section{RESULTS}

Five HIV-1 seropositive male patients died subsequent to participation in IVF-ICSI. The characteristics of these patients are summarized in Table 1.

The clinical course for the first couple was previously described. ${ }^{19}$ After three unsuccessful IVF cycles in Italy, the patient's wife at age 40, presented to Columbia University for participation in the program for HIV-1-serodiscordant couples. The husband, at age 36, suddenly died secondary to worsening pulmonary

Table 1. Characteristics of the HIV-Seropositive Men Who Died Subsequent to IVF-ICSI

\begin{tabular}{lcccccc}
\hline Subject & $\begin{array}{c}\text { Age } \\
\text { (years) }\end{array}$ & Race & $\begin{array}{c}\text { Duration of } \\
\text { HIV infection } \\
\text { (years) }\end{array}$ & $\begin{array}{c}\text { Viral load } \\
\text { (copies per millititer) }\end{array}$ & $\begin{array}{c}\text { CD4 T-cell } \\
\text { count } \\
\left(/ \mathrm{mm}^{3}\right)\end{array}$ & $\begin{array}{c}\text { Antiretroviral } \\
\text { Rx? }\end{array}$ \\
\hline 1 & 36 & Caucasian & 11 & 212 & 50 & Yes \\
2 & 45 & Caucasian & 17 & Undetectable & 624 & No \\
3 & 29 & Caucasian & 14 & 420 & 688 & Yes \\
4 & 33 & Caucasian & 18 & Undetectable & 639 & Yes \\
5 & 39 & Caucasian & 15 & Undetectable & 350 & Yes \\
\hline
\end{tabular}

IVF-ICSI, in vitro fertilization with intracytoplasmic sperm injection. 
hypertension shortly after the IVF-ICSI cycle. The wife did not achieve pregnancy with the fresh cycle, but returned 3 months later, seeking the transfer of her cryopreserved embryos. After consideration of the deceased's directives and the review by the Ethics Committee, an FET was performed successfully. A healthy, 3000-gram male child was delivered by cesarean section.

The second patient was 29 years old at initial consultation. He had a history of hemophilia and was coinfected with hepatitis $C$ and died secondary to complications of liver failure approximately 1 month after the birth of his children. The patient had a history of interferon treatment and was not noted to be viremic upon initiation of infertility treatment. The couple achieved pregnancy on the fourth cycle of IVF, when three embryos implanted. Three live-born infants were delivered via cesarean section at 30 weeks secondary to severe preeclampsia. All three were of very low birth rate, with the smallest one suffering an intraventricular hemorrhage. All children have thrived and are developing normally at 1 year of age.

The third patient presented at 33 years of age. This patient also had a history of hemophilia and hepatitis $C$ with a history of interferon treatment. The patient was not noted to be viremic by the assessment of the patient's primary care provider, nor did he suffer from any other active comorbidities at the initiation of infertility treatment. During the last few days of his wife's ovarian stimulation cycle, the patient became acutely ill and suddenly died on the day after oocyte aspiration. Postmortem examination determined aseptic meningitis as the cause of death. All day 3 embryos were cryopreserved. One year later, the surviving spouse renewed her agreement to continue storage of her frozen embryos, and has yet to decide whether or not she wishes to use them in the future.

The fourth patient was 45 years old with a history of azoospermia. The patient's wife was 43 years old with normal ovarian parameters. The couple attempted two cycles of IVF with donor sperm but failed to become pregnant. The patient died shortly thereafter secondary to intracranial hemorrhage.
The fifth patient was 37 years old when first presenting for care. He too was a hemophiliac coinfected with HIV-1 and hepatitis C, treated in the past with interferon. He and his wife were successful in both attempts at IVF, and delivered healthy children. Approximately 1 year after the birth of his second child, he suffered a severe contusion of the leg, leading to septicemia. Liver failure ensued and he died while awaiting a liver transplant. The wife and children remain well 2 years after his death.

No cases of HIV-1 seroconversion were detected in any of the female partners or offspring within the follow-up period (3 months postprocedure for nonpregnant patients, and 6 months postpartum for patients who conceived).

\section{DISCUSSION}

The deaths of these five patients in the short term after undergoing assisted reproduction underscores the tenuous situation of individuals with serious medical conditions who wish to start families. These patients were generally healthy and met criteria for entry into the program for HIV-1-serodiscordant couples. Three of the five patients had a significantly longer duration of infection compared to the general cohort of HIV-1-seropositive men in our program $(n=130,8.6$ years, \pm 5.5 ; range, $1-20)$. One patient had a CD4 count at the AIDS-defining level, but was stable and without a history of opportunistic infections according to the patient's infectious disease specialist, and was given clearance for entering the program. For all five patients, the HIV-RNA viral loads, a sensitive prognosticator of morbidity, ${ }^{20}$ were within the acceptable range for entry into the clinical program and were comparable to the general cohort of HIV positive men with detectable viral loads $(n=65,3660$ copies per milliliter, \pm 6338 ; range, 50-30,424), and were documented to be stable over the 6 months prior to beginning therapy. All five of these patients were Caucasian, as are most of the HIV-positive men in the cohort, and four of the five patients were on retroviral medications, also consistent with the trend in the cohort $(117 / 130$ on antiretroviral medications). The lack of obvious 
predictors for morbidity and mortality stresses the potentially unpredictable course of HIV infection even in clinically stable patients.

Offering assisted reproduction to HIV positive individuals remains controversial. The risks of viral transmission from the semen of HIV-positive men used for assisted reproduction warranted initial apprehension. In 1990, a female in Virginia seroconverted after unsuccessful insemination with her HIV-positive husband's sperm. While the events surrounding her infection remain controversial, the Centers for Disease Control cited this incident as a reason not to support the use of insemination of washed sperm as a means of reducing risk of HIV transmission. ${ }^{21}$ In the 15 years since that event, a large body of evidence has accumulated favoring assisted reproductive techniques, both sperm washing and IVF-ICSI, as safe and useful for allowing conception in serodiscordant couples. In the largest set of data reported from Europe, not a single transmission from an infected male to a female has occurred when assisted reproduction, primarily intrauterine insemination with washed sperm, was used. ${ }^{14}$ Similarly, in our cohort, which is significantly smaller than the European experience yet the largest in the United States, using IVF-ICSI not a single infection has occurred. With the infection rate for a single unprotected act of intercourse between a HIVpositive male and an uninfected female on the order of $0.1 \%-0.2 \%, 22,23$ it is possible that the current body of evidence is not powerful enough to prove that assisted reproduction is significantly safer than intercourse as a means of reducing the risk of HIV transmission. However, the cumulative data strongly suggests that assisted reproduction is effective and safe for HIV-discordant couples and may provide considerable risk reduction of viral transmission. The fetus is not at risk of infection as long as the female remains virus free, because transmission of HIV from a seronegative mother to her neonate has never been documented. Therefore, if assisted reproduction can be used to eliminate periconceptual transmission from a seropositive male to his seronegative partner, perinatal infection appears implausible.

The impetus for considering childbearing for HIV-positive individuals is the prolonged longevity that many patients now enjoy as a result of advances in antiretroviral therapy. With optimal management, the prospect for child rearing appears realistic. Given the recent statements that HIV-1 infection should not serve as a sole contraindication to assisted reproduction, $, 1,2$ in addition to mounting opinion in the field favoring fertility treatment for HIV-1-infected patients, ${ }^{10,24,25}$ biases against providing assisted reproduction to HIV-1-seropositive patients appear to be gradually weakening. A permissive attitude toward providing reproductive care to HIV-1-seropositive patients recognizes the autonomy of infected individuals, some of whom would assume risks to attempt to conceive naturally in the absence of reproductive assistance ${ }^{13}$; additionally, it protects the welfare of the uninfected partner and the offspring, who may be at a higher risk of infection if assisted reproductive services were not available. Compelling arguments have been made comparing HIV-1 infection to other chronic conditions that pose substantial threats to the health of the mother and child, yet are not categorical contraindications to assisted reproduction as HIV infection was classified in the recent past. ${ }^{10}$ In light of the optimistic trends in the management of HIV-1 and the favorable support for provision of fertility services, physicians may feel obligated to provide reproductive services to serodiscordant couples as they would to the general patient population. While we strongly advocate access to assisted reproductive services for HIV1-seropositive individuals and HIV-1-serodiscordant couples, as this report demonstrates, these patients have unique risks, and several important issues should be kept in mind when providing fertility care.

Assuming both partners are making voluntary decisions to participate, of foremost importance is the welfare of the offspring. Because the risks of infection to the fetus or neonate in the situation in which the father is seropositive and the mother is seronegative appears to be negligible, the pertinent issue then becomes the social welfare of a child born to a HIV-1-infected parent. The practical considerations are obvious and no different than those that confront any family, albeit possibly more pressing when a parent has a fatal disease. An acutely 
ill parent may not have the ability to simultaneously care for themselves and the child, thus provisions must be made for when sickness ensues. In a large survey of HIV-1-seropositive parents living with their children, $21 \%$ reported being hospitalized during the previous 6 months, including $10 \%$ who had been hospitalized for 1 week or more. ${ }^{26}$ Estate planning, and directives for guardianship are issues that potential HIV-1-serodiscordant individuals should address when making the decision for child bearing. In a survey of a subset of our HIV-1-serodiscordant population, who are not representative of the socioeconomic demography of the HIV-1-infected population in general, most couples addressed the possibility of raising a child alone in the event of a premature death of a spouse, but nearly $40 \%$ failed to address the possibility of third-party parenting, in the event of the demise of both parents. ${ }^{13}$ Any child of a parent with AIDS will certainly be affected by their parent's disease. The stigma associated with HIV-1 can hinder access to support services that could potentially be of assistance with coping. ${ }^{26}$ While such issues may be uncomfortable topics of discussion, when a couple presents for fertility consultation the conversation should be forthright, as not all patients have considered the possibilities.

Explicit advanced directives are imperative in reference to the use of cryopreserved gametes and embryos, and their potential posthumous use. Numerous ethical and legal issues surround the topic of posthumous reproduction, and legislature is variable with regards to the reproductive rights of the surviving partner, and survivorship entitlements of the resultant offspring. ${ }^{27}$ Conception using the genetic material of a deceased individual should only be entertained according to the explicit advanced directives of that person. ${ }^{28}$ Because not all patients who seek to start a family when they are alive wish for the same in death, explicit directives are of utmost importance to dictate the use of gametes or embryos posthumously. ${ }^{29}$ If the decision is duly made to proceed with posthumous reproduction, again, the regards for the welfare of the offspring are vital.

While the scope of this discussion and the bulk of our experience is limited to HIV-positive men with seronegative female partners, the treatment of HIV-positive women is also becoming increasingly frequent. Quoting the benefits of HAART and the dramatically reduced vertical transmission rates with current standards of care in pregnancy and labor, advocates assert that HIV-positive females should have full access to infertility services. ${ }^{30}$ When serodiscordant couples are involved in a stable relationship, it can be argued that the child will have the benefit of being raised by at least one healthy parent, thus it is less important which parent is HIV positive. In the situation where a HIV-positive woman is single and wishes to conceive without a partner, the risk that a child could be orphaned by AIDS is tenable, and such a scenario deserves thoughtful deliberation. To complicate matters further, because HIV superinfection can occur between two patients with primary infections, ${ }^{31}$ it can be argued that assisted reproduction may be indicated in couples in which both partners are HIV-infected to reduce the risk of introduction of resistant strains. The implications of HIVpositive parenting are obviously compounded when both parents are infected.

In conclusion, while we strongly advocate the access to fertility care for HIV-infected individuals, as the reported cases illustrate, serious issues must be considered when counseling these patients of the risks and benefits of assisted reproduction. While as the provider of fertility treatment, the primary goal is to allow a safe conception, the larger issues must not be ignored. Children of HIV-infected persons can be considered part of the HIV-affected population, ${ }^{26}$ thus the reproductive specialist potentially has a role in impacting the disease burden. Frank discussions and full disclosure are necessary to ensure that HIV-positive individuals can make informed, autonomous family planning decisions.

\section{REFERENCES}

1. Committee on Ethics of the American College of Obstetricians and Gynecologists. Human immunodeficiency virus: Physicians' responsibilities. ACOG Committee Opinion No. 255. April 2001.

2. Human immunodefieiency virus and infertility treatment. Fertil Steril. 2004;82(Suppl 1):S228-231.

3. Ethics Committee of the American Society for Reproductive Medicine. Human immunodeficiency virus 
infection: Physicians' responsibilities. Committee on Ethics, The American College of Obstetricians and Gynecologists. Obstet Gynecol 1990;75:10431045.

4. Ethical considerations of assisted reproductive technologies. By the Ethics Committee of the American Fertility Society. Fertil Steril 1994;62(5 Suppl 1):1S125 S.

5. Grossman M. Human immunodeficiency virus infections in children: Public health and public policy issues. Pediatr Infect Dis J 1987;6:113-116.

6. Rizk B, Dill SR. Counselling HIV patients pursuing infertility investigation and treatment. Hum Reprod 1997;12:415-416.

7. Schenker JG. FIGO Committee for the Study of Ethical Aspects of Human Reproduction: guidelines on the subject of AIDS and human reproduction. Hum Reprod 1997;12:1619.

8. Smith JR, Forster GE, Kitchen VS, Hooi YS, Munday $\mathrm{PE}$, Paintin DB. Infertility management in HIV positive couples: A dilemma. BMJ 1991;302:1447-1450.

9. From the Centers for Disease Control and Prevention. HIV and AIDS-United States, 1981-2000. JAMA 2001;285:3083-3084.

10. Gilling-Smith C, Smith JR, Semprini AE. HIV and infertility: Time to treat. There's no justification for denying treatment to parents who are HIV positive. BMJ 2001;322:566-567.

11. Fauci AS, Pantaleo G, Stanley S, Weissman D. Immunopathogenic mechanisms of HIV infection. Ann Intern Med 1996;124:654-663.

12. Chen JL, Philips KA, Kanouse DE, Collins RL, Miu A. Fertility desires and intentions of HIV-positive men and women. Fam Plann Perspect 2001;33:144-152, 165.

13. Klein J, Pena JE, Thornton MH, Sauer MV. Understanding the motivations, concerns, and desires of human immunodeficiency virus 1-serodiscordant couples wishing to have children through assisted reproduction. Obstet Gynecol 2003;101(5 Pt 1):987-994.

14. Semprini AE, Fiore S. HIV and reproduction. Curr Opin Obstet Gynecol 2004;16:257-262.

15. Stern JE, Cramer CP, Garrod A, Green RM. Access to services at assisted reproductive technology clinics: A survey of policies and practices. Am J Obstet Gynecol 2001;184:591-597.

16. Sauer MV, Chang PL. Establishing a clinical program for human immunodeficiency virus 1- seropositive men to father seronegative children by means of in vitro fertilization with intracytoplasmic sperm injection. Am J Obstet Gynecol 2002;186:627-633.

17. Steyaert SR, Leroux-Roels GG, Dhont M. Infections in IVF: review and guidelines. Hum Reprod Update 2000;6:432-441.

18. Palermo GD, Cohen J, Alikani M, Adler A, Rosenwaks Z. Development and implementation of intracyto- plasmic sperm injection (ICSI). Reprod Fertil Dev 1995;7:211-217.

19. Sauer MV, Chang PL. Posthumous reproduction in a human immunodeficiency virus-discordant couple. Am J Obstet Gynecol 2001;185:252-253.

20. Tarwater PM, Gallant JE, Mellors JW, et al. Prognostic value of plasma HIV RNA among highly active antiretroviral therapy users. AIDS 2004;18:2419-2423.

21. HIV-1 infection and artificial insemination with processed semen. MMWR Morb Mortal Wkly Rep 1990;39:249, 255-246.

22. de Vincenzi I. A longitudinal study of human immunodeficiency virus transmission by heterosexual partners. European Study Group on Heterosexual Transmission of HIV. N Engl J Med 1994;331:341-346.

23. Mastro TD, de Vincenzi I. Probabilities of sexual HIV1 transmission. AIDS 1996;10(Suppl A):S75-82.

24. Englert Y, Van Vooren JP, Place I, Liesnard C, Laruelle C, Delbaere A. ART in HIV-infected couples: Has the time come for a change of attitude? Hum Reprod 2001;16:1309-1315.

25. Spriggs M, Charles T. Should HIV discordant couples have access to assisted reproductive technologies? J Med Ethics 2003;29:325-329.

26. Schuster MA, Kanouse DE, Morton SC, et al. HIV-infected parents and their children in the United States. Am J Public Health 2000;90:1074-1081.

27. Land S, Ross LS. Posthumous reproduction: current and future status. Urol Clin North Am 2002;29: 863-871.

28. Ethics Committee of the American Society for Reproductive Medicine. Posthumous reproduction. Fertil Steril 2004;82(Suppl 1):S260-262.

29. Brenwald ML, Redeker K. A primer on posthumous conception and related issues of assisted reproduction. Washburn Law J 1999;38:599-654.

30. Minkoff H, Santoro N. Ethical considerations in the treatment of infertility in women with human immunodeficiency virus infection. N Engl J Med 2000;342: 1748-1750.

31. Chan DJ. HIV-1 superinfection: Evidence and impact. Curr HIV Res 2004;2:271-274.

Address reprint requests to: Mark V. Sauer, M.D.

Director, Division of Reproductive Endocrinology and Infertility Center for Women's Reproductive Care at Columbia University 1790 Broadway, 2nd Floor New York, NY 10019

E-mail:mvs9@columbia.edu 